\title{
The relationship between spatial resolution levels and quantitative myocardial perfusion
}

\author{
Niloufar Zarinabad ${ }^{1 *}$, Gilion Hautvast ${ }^{4}$, Marcel Breeuwer ${ }^{2,3}$, Eike Nagel ${ }^{1}$, Amedeo Chiribiri $^{1}$ \\ From 16th Annual SCMR Scientific Sessions \\ San Francisco, CA, USA. 31 January - 3 February 2013
}

\section{Background}

Dynamic contrast enhanced cardiovascular magnetic resonance imaging (DCE-CMR) allows for high-resolution analysis of myocardial perfusion by preserving important spatial information. However high resolution perfusion quantification suffers from the poor signal-to-noise ratio (SNR) of voxel-based data. Previously spatial averaging (segmental or sub-segmental analysis) has been used to improve the SNR. This approach results in reduction of spatial resolution and potentially in a loss of information about the extent, localization and transmurality of ischemia.

This study investigates the relationship between the level of spatial resolution and accuracy of perfusion estimates obtained using different deconvolution methods including Fermi function modelling, ARMA, B-spline basis and exponential basis deconvolution. A new quality of fit analysis method which uses autocorrelation function properties [1] is used here to measure the fraction of remaining un-modelled information (FRI) of the data (i.e. the amount of diagnostic information lost).

\section{Methods}

Perfusion data were obtained from five patients with a dual-bolus technique and $0.075 \mathrm{mmol} / \mathrm{kg}$ Gadobutrol (Gadovist, Bayer, Germany) injected at $4 \mathrm{ml} /$ minute followed by a $20 \mathrm{ml}$ saline flush using a Philips Achieva 3T (TX) system, equipped with a 32-channel cardiac phased array receiver coil (Philips, Best, Netherlands). Images were acquired using a saturation recovery gradient echo method (TR/TE $3.0 \mathrm{~ms} / 1.0 \mathrm{~ms}$, flip-angle $15^{\circ}$; effective k-t SENSE acceleration 3.8, spatial resolution $1.2 \times 1.2 \times 10 \mathrm{~mm}$, saturation-recovery delay $120 \mathrm{~ms}$ ).

'Division of Imaging Science and Biomedical Engeering, Kings College London, London, UK

Full list of author information is available at the end of the article
First, quantification was performed on high-resolution voxel based data. Then spatial averaging was performed in both transmural and angular direction. In each direction, the total number of grouped voxels (region of interest size) was increased to reduce spatial resolution to $50 \%, 20 \%$ and $10 \%$ of its original value. Analysis of the quality of the fitting procedure was performed on each stage of the process and the obtained results were compared.

\section{Results}

The average FRI increased as the resolution was reduced (Fig 1), particularly in the transmural direction. This indicates that increasing spatial averaging is responsible for an increase of the amount of unused information (FRI). This was maintained amongst all deconvolution methods and, in accordance with the expected subendocardial physiological distribution of perfusion defects, was particularly important for spatial averaging in the transmural direction.

\section{Conclusions}

Whilst a reduction in spatial resolution level increases SNR and enables a more stable quantification, a degree of physiological information loss is incurred. Angular direction averaging preserves more information in comparison to transmural averaging preserving potentially useful clinical CMR data.

\section{Funding}

The authors acknowledge financial support from the Department of Health via the National Institute for Health Research (NIHR) comprehensive Biomedical Research Centre award to Guys and St Thomas NHS Foundation Trust in partnership with Kings College London. 


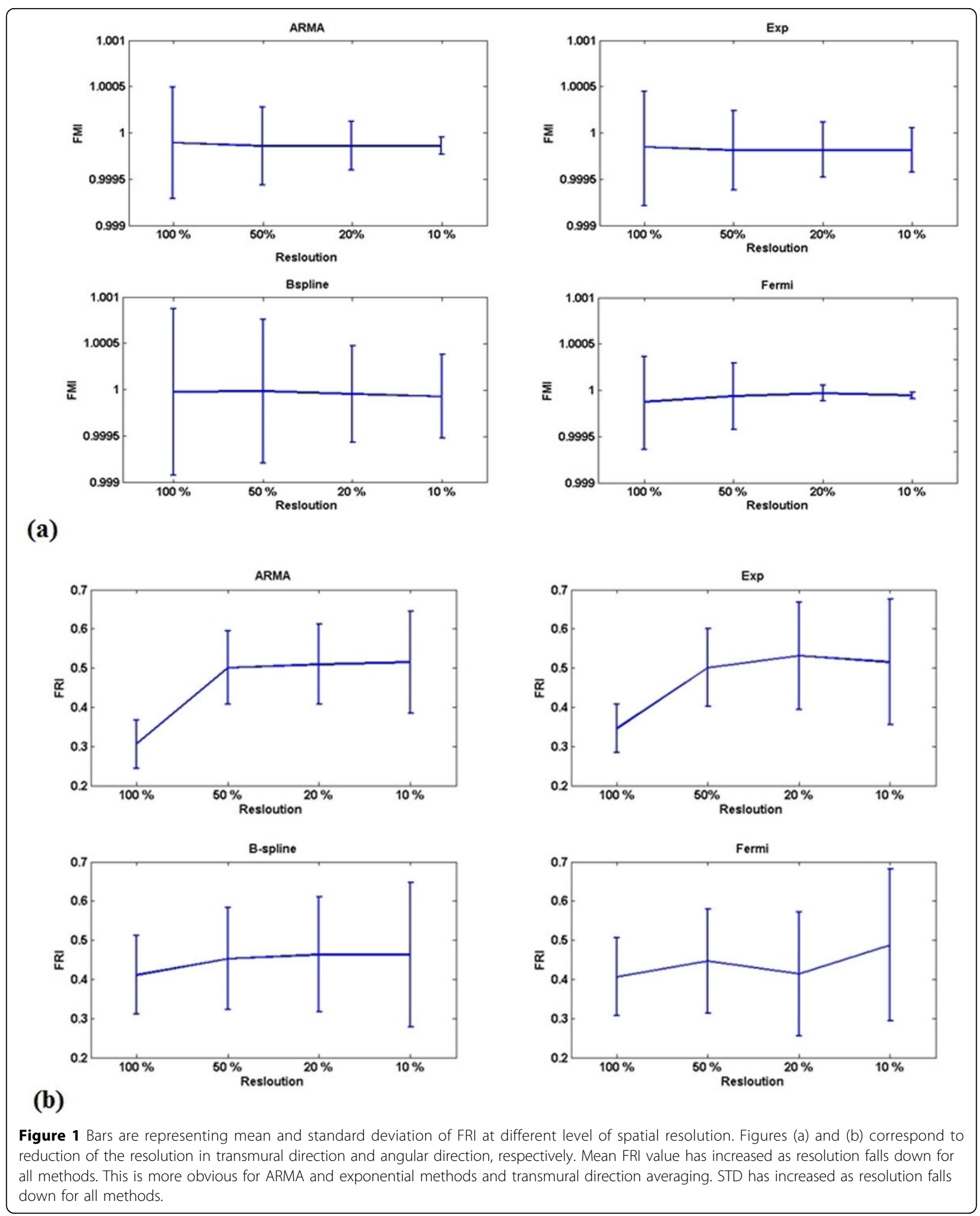




\section{Author details}

'Division of Imaging Science and Biomedical Engeering, Kings College London, London, UK. ${ }^{2}$ Biomedical Engineering,Biomedical Image Analysis, Eindhoven University of Technology, Best, Netherlands. ${ }^{3}$ Imaging Systems, MR, Philips Healthcare, Best, Netherlands. ${ }^{4}$ Healthcare Incubators, Philips Innovation Group, Eindhoven, Netherlands.

Published: 30 January 2013

\section{Reference}

1. Balvay D:. Mag Res Med 2005.

doi:10.1186/1532-429X-15-S1-084

Cite this article as: Zarinabad et al:: The relationship between spatial resolution levels and quantitative myocardial perfusion. Journal of

Cardiovascular Magnetic Resonance 2013 15(Suppl 1):O84.

Submit your next manuscript to BioMed Central and take full advantage of:

- Convenient online submission

- Thorough peer review

- No space constraints or color figure charges

- Immediate publication on acceptance

- Inclusion in PubMed, CAS, Scopus and Google Scholar

- Research which is freely available for redistribution

Submit your manuscript at www.biomedcentral.com/submit 